\title{
Altered branching ratios and relaxation oscillations in superfluorescent cascades
}

\author{
W A Molander and C R Stroud Jr \\ The Institute of Optics, University of Rochester, Rochester, NY 14627, USA
}

Received 13 July 1981, in final form 9 March 1982

\begin{abstract}
A non-linear rate equation describing superfluorescence in many-level systems is presented. It is shown that when a large atomic population is excited into Rydberg levels the cascade back to lower levels by cooperative spontaneous emission leads to a number of new effects including 'trapping' of population in excited states, alteration of branching ratios, truncation of superfluorescent pulses and multiple pulsing of a single transition.
\end{abstract}

When a collection of atoms is excited by a laser to a Ryberg state, it will cascade back down through a series of levels to the ground state. Due to the long wavelengths and large dipole moments of transitions between Rydberg states, the decaying atoms may be strongly coupled to each other by their common near field. Superfluorescence which can result from this strong coupling has been observed from Rydberg atoms by several groups. Gross et al (1979) in particular have emphasised the strength of the atom-atom coupling by observing superfluorescence in samples of only $10^{5}$ excited atoms. In this paper we show that superfluorescence can dramatically alter branching ratios and even the fundamental dynamics of these multi-level cascades. We find that transitions with the largest dipole moments are greatly enhanced so that substantially all the population goes down one channel without branching. We also find that the superfluorescence can be quenched by coherent trapping of the population in an excited level, but can subsequently be reinitiated, leading to a relaxation oscillation decay.

The analysis of this cascade is inherently a multi-level problem with the possibility of successive cooperative decays. However, the extensive literature of superfluorescence is almost completely devoted to the study of two-level systems. Agarwal and Trivedi (1976) and Ito (1976) have considered the three-level atom in the small-sample approximation. Ikeda (1977) discusses the swept excitation model for a three-level system. Crubellier et al (1980) have discussed both superradiance and subradiance in three-level atoms. Senitzky (1974) has derived the equations of small-sample multi-level superfluorescence from a boson second-quantisation formalism. However, none of these papers discuss the way in which superfluorescence changes branching ratios and the dynamics of cascades in a fundamental way.

In this paper we present an intuitively appealing non-linear rate equation which we have derived from source-field quantum electrodynamics. Since the derivation is analogous to a number of two-level calculations including that in the textbook of Allen and Eberly (1975), details of the derivation are not given here. Only the necessary assumptions and approximations are pointed out. Our chief aim here will 
be to examine a series of special cases and demonstrate a number of new phenomena which occur in multi-level superfluorescence, but not in two-level superfluorescence.

It is easily possible to prepare a system with a large excited-state population whose total dimensions are small compared with the wavelengths of the far-infrared transitions between the Rydberg states. Our theory will model this situation. We will assume that the electric field is uniform across the sample. Friedberg and Hartmann (1974) and Żakowicz (1978) have studied superfluorescence in these small systems and shown that the strong dipole-dipole interactions will have a tendency to dephase the dipoles as well as cause them to decay cooperatively. They have not treated multi-level systems. Nor have they considered low-density systems in which the random locations of the atoms may also affect the uniform-field assumption. However, from their model they conclude that for approximately spherical systems with a diameter smaller than a wavelength, the dephasing will not prevent the cooperative decay of a substantial part of the inversion.

Using Heisenberg-picture quantum electrodynamics (Allen and Eberly 1975) we can determine the equation of motion for the sum of the individual-atom density matrix elements

$$
R_{\alpha \beta}(t)=\sum_{i=1}^{N}\left\langle\sigma_{\alpha \beta}^{i}(t)\right\rangle
$$

The $\alpha$ and $\beta$ refer to the atomic energy levels while the index $i$ runs over the $N$ identical atoms. In the regime in which there is a macroscopic polarisation we can make the semiclassical approximation and factor the expectation values of the product of two operators referring to two atoms into the product of their expectations, i.e.

$$
\left\langle\sigma_{\alpha \beta}^{i}(t) \sigma_{\delta \gamma}^{j}(t)\right\rangle=\left\langle\sigma_{\alpha \beta}^{i}(t)\right\rangle\left\langle\sigma_{\delta \gamma}^{j}(t)\right\rangle \quad i \neq j .
$$

If, however, one of the transitions is almost totally inverted, no macroscopic polarisation will exist at that transition frequency. This situation occurs at $t=0$ and, in addition, can occur as we shall see, at subsequent times in the decay. However, even when there is no macroscopic polarisation each atom continues to decay due to its own radiation reaction field. Glauber and Haake (1978) have shown how these independent radiators will, after a delay time, become phased generating a macroscopic polarisation. The essential features of this quantum initiation can be taken into account by simply grafting linear spontaneous emission terms onto the non-linear cooperative decay equations. Though this procedure ignores the statistical nature of the initiation it can be shown to give correct results for expectation values. These considerations lead to the following equation,

$$
\dot{R}_{n n}=\sum_{E_{p}>E_{n}} \gamma_{p n}\left(R_{p n} R_{n p}+R_{p p}\right)-\sum_{\substack{p \\ E_{p}<E_{n}}} \gamma_{n p}\left(R_{p n} R_{n p}+R_{n n}\right) .
$$

The $\gamma$ are just Einstein $A$ coefficients.

This equation is easily understood. The quadratic terms are just the square of the expectation of the macroscopic dipole moment. This is just the classical radiation rate for the macroscopic collective polarisation associated with each transition. The linear terms are just the sum of the single-atom spontaneous decay terms.

This equation can be greatly simplified if we make one further assumption-that every atom has the same initial condition. This is quite reasonable for a small sample excited by a laser pulse. A group of atoms each initially in the same pure state, each 
acted on by the same coherent field will each evolve in the same way to another pure state. In our case the atoms are all subject to the same coherent field except when some transition is nearly totally inverted. However, we have already pointed out that the quantum initiation will cause one mode of oscillation to grow and phase all of the atomic dipoles together. Since this phasing occurs before any substantial decay from the excited state, the atoms will all be in the same pure state during the entire decay. When this is the case it is easy to show that

$$
\boldsymbol{R}_{p n} \boldsymbol{R}_{n p}=\boldsymbol{R}_{p p} \boldsymbol{R}_{n n}
$$

the square of the dipole moment is equal to the product of the populations in the upper and lower levels of the transition. With this change our equation of motion becomes

$$
\dot{R}_{n n}=\sum_{\substack{p \\ E_{p}>E_{n}}} \gamma_{p n} R_{p p}\left(R_{n n}+1\right)-\sum_{\substack{p \\ E_{p}<E_{n}}} \gamma_{n p} R_{n n}\left(R_{p p}+1\right) .
$$

This non-linear rate equation is our principal result. This same equation (without the linear decay terms) was derived to describe spontaneous emission of a single atom in the neoclassical theory by Crisp and Jaynes (1969). It was also obtained by Senitzky (1974) under assumptions similar to those used here but using a boson second quantisation formalism. The equation shows that cooperative decay in a multi-level system has a rate proportional to the product of the populations in the two states linked by a dipole transition. The cooperative decay will stop if there is no population in the lower state, and it will be enhanced if population is fed into the lower state.

For general $\gamma$ this equation is too difficult to solve analytically for anything more complex than a two-level system. We can obtain an intuitive understanding adequate for discussing even very complicated systems by studying a few special cases however.

First, let us review the two-level solutions. The inversion decays as a hyperbolic tangent. There is no metastability. The linear terms cause decay even when there is no macroscopic polarisation.

If we go to the next more complicated system, a three-level cascade, illustrated in figure 1, we find results may be just two successive two-level decays, or something very different. In figure $1(b)$ we have taken $\gamma_{12}=10 \gamma_{23}$. The first transition takes place very quickly before the second can start, then later the second follows. This is just two successive two-level decays. However, in figure $1(c)$ we see the results when $\gamma_{12}=\frac{1}{10} \gamma_{23}$. In that case the decay begins slowly. As population begins to build up in the intermediate state the second transition quickly takes off and depletes the population in the intermediate state. Population can never accumulate again in the intermediate state so there can be no further cooperative decay from level 1. Population which is trapped there can be dissipated only by incoherent decay (Ito 1976, Ikeda 1977).

Cascades generally involve a complicated branching and rejoining of many paths down the ladder of states. The non-linear equations can treat that as well. In figure 2 we see illustrated the case where the cascade branches from a common upper state to two lower states. If we take $\gamma_{12}=\frac{2}{3} \gamma_{23}$ we do not find the branching ratio to be $2: 3$ as expected from single-atom decay. Instead the preferred channel's dominance is enhanced by the non-linear decay. In this example with $N=10^{5}$ the branching ratio is approximately $1: 46$. For larger $N$ the enhancement is larger.

A third situation involving three levels which occurs in a cascade is the joining of population from two upper levels to a common lower level. This is illustrated in 

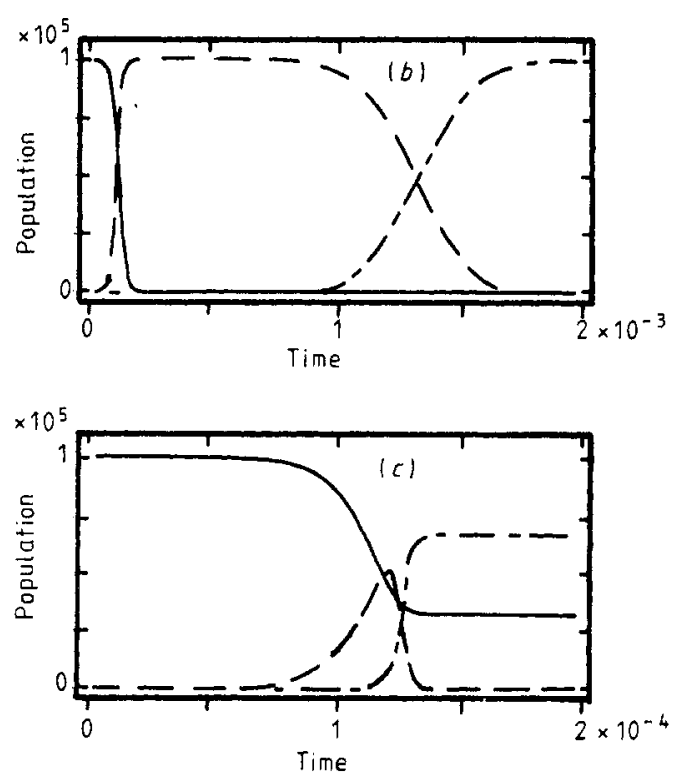

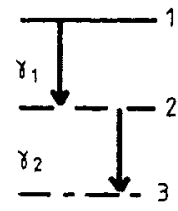

(a)

Figure 1. (a) The energy level diagram for the three-level cascade with the levels designated by the symbols used in the plots. $(b)$ The populations of the three levels as a function of time. Here and in all the examples the number of atoms is $10^{5}$ and the time is in units of $\gamma_{1}^{-1}$. In this plot $\gamma_{2}=\frac{1}{10} \gamma_{1}$. (c) The populations of the three levels as a function of time. $\gamma_{2}=10 \gamma_{1}$. The rapid second decay quenches the first transition trapping population in level 1 .
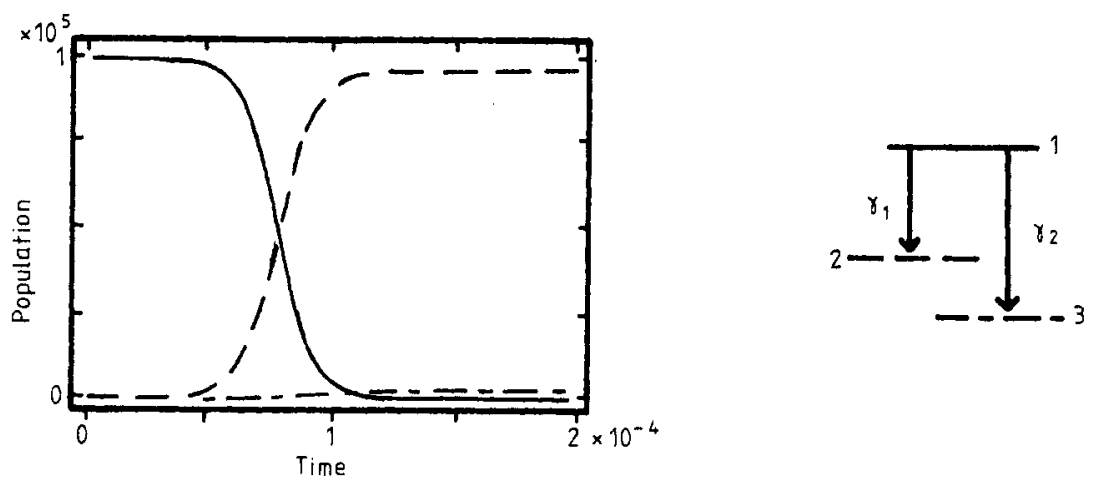

Figure 2. The populations of the three levels in a $\lambda$ system as a function of time. $\gamma_{2}=\frac{3}{2} \gamma_{1}$ so the linear branching ratio is $2: 3$ but the non-linearities make it about $1: 46$ in the cooperative system.

figure 3. Here we have taken the two decay rates to differ by a factor of 10 , and have taken the initial population to be equally distributed between the two excited states. The result is that the fast transition starts increasing the population in the common lower level. This increases the rate of the slower transition by increasing the product of populations. Thus, the fast transition also speeds up all transitions having a common lower state. The cooperative enhancement of the slower transition by the faster one assumes that each transition is described by a coherent microscopic polarisation, and thus there is coherence between the two upper levels. Again this will be true if each 

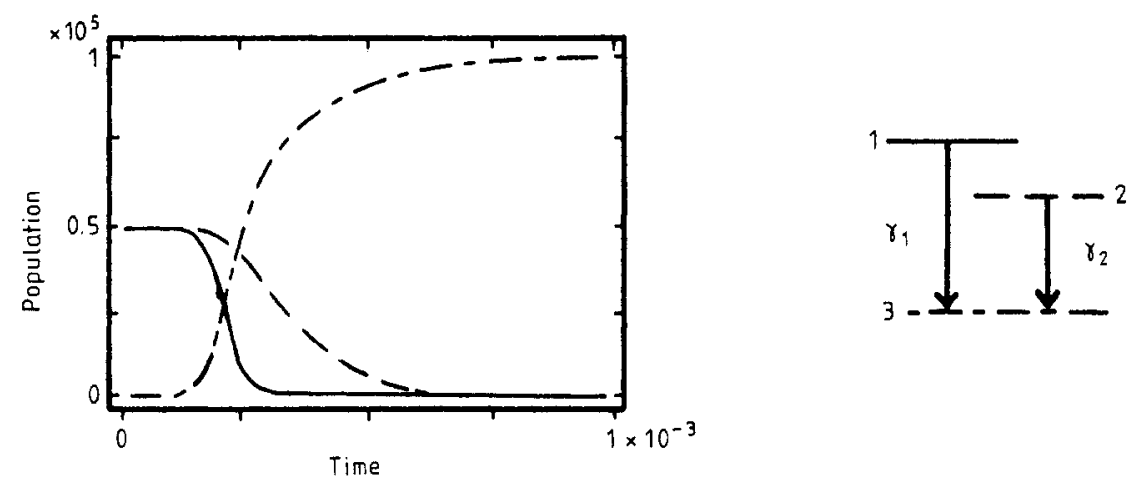

Figure 3. The populations of the three levels in a V system as a function of time, $\gamma_{2}=\frac{1}{10} \gamma_{1}$. The population is initially equally divided between the excited states. The 2-3 transition occurs almost as fast as the 1-3 transition because the latter feeds population into the lower state.

of the upper levels was populated by superfluorescent transitions from above. Crubellier et al (1980) have found that coherent trapping can occur in this system if the initial coherence is not complete.

Finally, we will look at a four-level cascade to see what happens in this more complex case. This is shown in figure 4. Here we have made the second transition
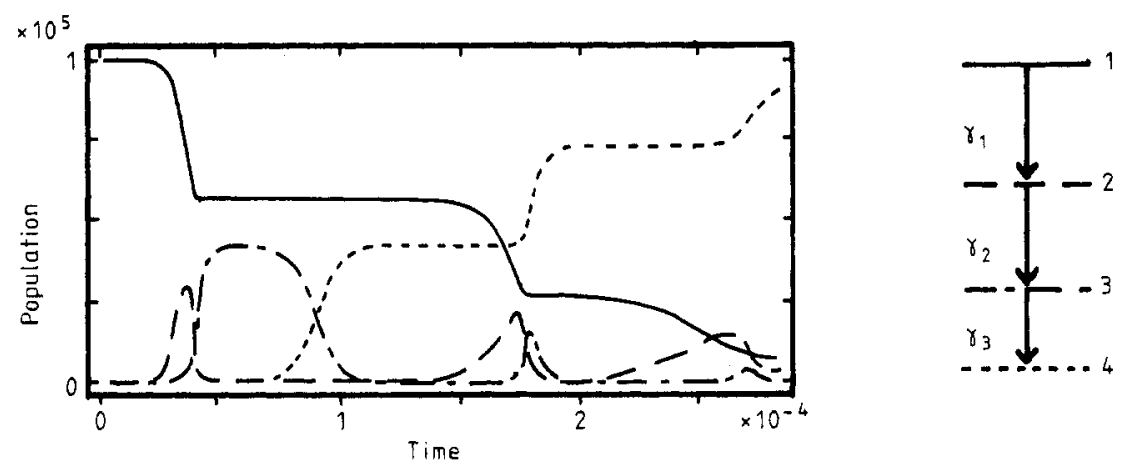

Figure 4. The populations of the four levels in a cascade as a function of time. $\gamma_{2}=\gamma_{3}=$ $1.8 \gamma_{1}$. The population in each of the intermediate levels pulses due to repetitive generation and quenching of the non-linear decay.

more rapid than either the upper or lower transitions. At the beginning the situation is just that which we saw in figure $1(c)$. The first transition begins but is truncated as the second rapid transition depletes the population in level 2. The first three levels are then returned to their initial state except the population in level 1 is somewhat less than it was originally. The process then begins again with a slightly slower non-linear damping than before. Again the decay will be truncated, etc. We will get multiple pulsing of the transitions until the population is reduced below the threshold for cooperative decay. A general rule emerges which summarises some of these observations. The non-linear competition between transitions tends to direct all the population down the most favoured branch. 
These rather surprising effects of preferred decay channels and relaxation oscillations were derived under restrictive assumptions, namely small-sample theory. However, the effects are quite general, dependent only on the non-linear nature of the basic equations, thus it seems quite reasonable to assume that they will be manifest even when dipole-dipole and propagation effects are included.

\section{Acknowledgments}

We would like to acknowledge partial support of this work by the Air Force Office of Scientific Research, the Army Research Office, the Science Research Council (Great Britain), and the Hertz Foundation. C R Stroud would like to thank L Allen for helpful discussions.

\section{References}

Agarwal G S and Trivedi S S 1976 Opt. Commun. $18417-20$

Allen L and Eberly J H 1975 Optical Resonance and Two-Level Atoms (New York: Wiley) ch 8 Crisp and Jaynes 1969

Crubellier A, Liberman S and Pillet P 1980 Opt. Commun 33 143-8

Friedberg R and Hartmann S R 1974 Phys. Rev. A 10 1728-39

Glauber R and Haake F 1978 Phys. Lett. 68A 29-32

Gross M, Goy P, Fabre C, Haroche S and Raimond J M 1979 J, Physique 43 343-6

Ikeda K 1977 Phys. Lett. 63A 248-50

Ito H 1976 Prog. Theor. Phys. 55 1038-48

Senitzky I R 1974 Phys. Rev. A 10 1868-73

Zakowicz W 1978 Phys. Rev. A 17 343-52 